Pathologe 2010 - [Suppl 2] 31: 116-118

DOI 10.1007/s00292-010-1315-0

Online publiziert: 22 . Juli 2010

๑) Springer-Verlag 2010
H.H. Kreipe

Institut für Pathologie, Medizinische Hochschule Hannover

\title{
Eröffnungsrede des Tagungs- präsidenten der Deutschen Gesellschaft für Pathologie e.V.
}

Sehr geehrte Damen und Herren, liebe Kolleginnen und Kollegen!

Ich danke Frau Schmitt-Gräff und Herrn Schlake für die Begrüßungsworte zu unserer gemeinsamen Woche der Pathologie, die zum dritten Mal abgehalten wird. Sie soll vor Augen führen, dass unser Fach bei allen natürlichen Egoismen der drei Verbände, am besten daran tut, wenn gemeinsam vorgegangen wird. Meine Erfahrung aus den letzten neun Jahren Vorstandsarbeit in der Deutschen Gesellschaft für Pathologie (DGP) ist, dass dies nicht immer einfach, manchmal sogar sehr schwer, aber immer notwendig und im Interesse unseres Faches ist. Die erforderliche Arbeitsteilung zwischen wissenschaftlichem Austausch, Fortbildung und berufsständischer Vertretung, die wir als stark in die Krankenversorgung eingebundene, auf nebenberufliches Engagement angewiesene kleine Berufsgruppe vornehmen müssen, darf nicht zu kurzsichtiger Kleinstaaterei der drei Verbände DGP, BV (Bundesverband Deutscher Pathologen) und IAP (Internationale Akademie für Pathologie) führen. Denn die Herausforderungen, die vor uns liegen sind erheblich und nur gemeinsam zu bestehen.

Molekulare Tumorpathologie repräsentiert eines der drei Schwerpunktthemen der 94. Jahrestagung am Beginn eines Jahrzehnts, in dessen Verlauf die Pathologie ihr Gesicht verändern wird. Wir stehen an einem immer deutlicher werdenden Wendepunkt, und die nächsten 10 Jahre werden darüber entscheiden, ob die traditionell starke Position der Pathologie in der Tumordiagnostik bestehen bleibt.

Dass Tumoren als genetisch bedingte Erkrankungen auch anhand der zugrunde liegenden genetischen Defekte erkannt und klassifiziert werden, erscheint naheliegend und mittelfristig unumgänglich. Dabei hat die Zukunft bereits begonnen. Am Samstag wird Dr. Frederik Baehner, der verantwortliche Pathologe von $\mathrm{Ge}$ nomic Health, im Rahmen eines Satellitensymposiums die auf RNA-Expressionsprofile beruhenden prognostischen Scores beim Mamma- und Kolonkarzinom vorstellen. In den USA werden mittlerweile 60\% aller Östrogenrezeptor-positiven, nodalnegativen Mammakarzinome mit Hilfe des „Recurrence-Score“ molekular graduiert.

In zwei großen Studien, TAILORx und MINDACT, mit jeweils mehr als 6000 Mammakarzinompatientinnen wird zurzeit prospektiv evaluiert, inwieweit sich durch präzisere Prognostik Chemotherapie vermeiden lässt. Die Fragestellung dieser Studien ist zwingend, sind doch mehr als $70 \%$ der Mammakarzinompatientinnen durch die Operation allein geheilt und bedürften keiner weiteren Therapie, die aber mehr als $90 \%$ erhalten, da die traditionellen pathologisch-anatomischen Parameter nicht sicher genug erscheinen.

Die Histopathologie ist gut und sicher bei der Dignitätsfrage, doch zu unpräzise und zu wenig reproduzierbar bei der immer wichtigeren Frage der Graduierung. Diese Frage gewinnt nicht nur an Bedeutung wegen der Zunahme der therapeu- tischen Optionen mit individualisierter und risikoadaptierter Indikationsstellung, sondern auch, weil uns Screening-Verfahren wie PSA und Mammographie mehr und mehr Tumoren bescheren, die histologisch bösartig, biologisch aber unter Berücksichtigung der Lebenszeitperspektive eher harmlos sind. Ohne zusätzliche neue und molekulare Marker werden wir bei vielen Tumoren entscheidende Antworten schuldig bleiben.

An dieser Stelle fallen stets zwei Einwände der Bedrohungsrhetorik ins Wort. Der beschwichtigenden Frage, was denn von den vielen und hochrangig publizierten Genexpressionsarrays hämatopathologischer Tumoren, dem zweiten Schwerpunktthema dieser Tagung, tatsächlich in die Diagnostik eingeflossen sei, ist aber leicht zu begegnen. Gerade bei diesen Neoplasien, einigen Lymphomen, besonders aber der akuten myeloischen Leukämie und den chronischen myeloproliferativen Neoplasien zeigt sich, wie sich anhand einer handvoll genetisch definierbarer klonaler Defekte eine deutlich verbesserte Klassifizierung und prognostische Stratifizierung erreichen lässt. Hier gehört die molekularpathologische Analyse bereits zum Standard. Der gröberen morphologischen Vorsortierung folgt die feinere molekulare Analyse.

Der zweite Einwand stellt die Frage, ob mit den molekularen Methoden nicht mit viel größerem Aufwand bestimmt wird, was auch durch optimierte histopathologische Befundung und Immunhistochemie zu erreichen sei. In der Tat scheinen der Recurrence-Score und der Agendia- 
70-Genscore beim Mammakarzinom im Wesentlichen die Proliferation der Tumorzellen wider zu spiegeln, die immunhistochemisch leicht zu erfassen ist. Paradoxerweise erfahren über den Umweg der Genexpressionsarrays immunhistochemische Marker wie Ki-67 bei der Definition vom Luminal-B-Typ des Mammakarzinoms und die Hormonrezeptoren beim triple-negativen bzw. basalen Phänotyp eine Renaissance als Klassifikatoren, was aber - da sollten wir uns nicht in die Irre führen lassen - dem Zukunftspotenzial der molekularen Graduierung keinerlei Abbruch tut.

Sicher, die Immunhistochemie ist eine potente, uns vertraute und den anatomischen Kontext bewahrende Technik. Ob sie sich aber gegen molekulare Techniken bei Prognostik und Prädiktion behaupten kann, hängt vor allem davon $\mathrm{ab}$, ob sie hinsichtlich des Treffens von Schwellenwerten ähnlich reproduzierbar und zuverlässig sein kann wie die Messung von Nukleinsäuren.

Wie wichtig Reproduzierbarkeit und Standardisierung zur Erhöhung der Sicherheit in der Medizin sind, kann man mit der Ausbreitung des Kaiserschnitts verdeutlichen, den die meisten Frauen nicht wollen, aber $20-30 \%$ erhalten, weil die traditionelle Zangengeburt einfach zu stark vom individuellen Können des einzelnen Geburtshelfers abhängt und ein, wenn auch minimales, aber höhere Restrisiko eines Fehlers aufweist.

Anders als die Geburtshelfer sind wir in der Pathologie glücklicherweise nicht in der Situation, dass sich die beiden Möglichkeiten ausschließen - sondern sie ergänzen sich. Daher stellt das rapide wachsende Gewicht der Molekularpathologie keine Bedrohung, sondern eine Chance für unser Fach dar, wenn es uns gelingt, versierte Histopathologie mit professioneller Molekularpathologie zu verbinden. So wie der Chirurg mit „der Bauch gehört mir" keinen Zweifel an seiner monopolistischen Zuständigkeit zulässt, sollten wir keinen Zweifel daran zulassen, dass die morphologische und molekulare Klassifikation von Tumoren im soliden Gewebe Aufgabe der Pathologie ist.

Diesen Anspruch zu erheben, bedeutet allerdings nicht, dass er damit allein auch gewahrt ist. Entscheidend für seine Wah- rung ist, dass Expertise im Fach entsteht und ausgebaut wird, dass eine neue Generation von Pathologinnen und Pathologen heranwächst, denen der tägliche Brückenschlag zwischen morphologischem und molekularem Befund bei der Klassifikation von Tumoren zur Routine wird. Gelingen wird das nur dem, der mit eigenen wissenschaftlichen Projekten am Fortschritt teilgenommen und dabei genuine molekularpathologische Methodenerfahrung gesammelt hat.

Vor diesem Hintergrund stimmt es sehr optimistisch, wie viele Beiträge $\mathrm{zu}$ dieser Jahrestagung angemeldet wurden. Die Schrittmacherfunktion, die die Deutsche Pathologie bei den neuen prädiktiven Biomarkern wie K-ras und EGFR in Europa eingenommen hat, ist vor allem aus ihrer Forschungsaktivität und der damit zusammenhängenden personellen und methodischen Infrastruktur zu erklären. Zu dem Selbstverständnis eines Universitätsinstitutes sollte es gehören, diese Forschungsinfrastruktur zu garantieren. In Zukunft wird gelten, dass nicht mehr ein breites und großvolumiges Eingangsspektrum das Kennzeichnende eines Universitätsinstitutes sein kann, sondern eine in Teilbereichen hoch spezialisierte morphologische und molekulare Diagnostik verbunden mit signifikanter Forschungsaktivität. Nur durch letztere wird methodische und medizinische Kompetenz erzeugt, die den zuvor beschriebenen Anspruch sichern und vor der Abdrängung in ein umgrenztes und steriles Reservat, in dem sich die Zytopathologie bereits befindet, bewahren kann. Selbstverständlich wollen wir auch in Zukunft die gesamte Breite des Faches an den Universitätsinstituten vertreten sehen, aber welche Volumina dazu erforderlich sind, muss mit einem Fragezeichen versehen werden.

Fragen müssen wir uns auch, ob der alles beherrschende singuläre Facharzt oder ein Team mit Teilspezialisierungen hinter unseren Weiterbildungskonzepten steht. Sollten die Weiterbildungsinhalte nicht klarer definiert und stärker methodisch ausgerichtet werden, statt sie in der „Rush-hour“ des Lebens mit Inhalten zu überfrachten, die die Forschungsaktivität strangulieren? Muss der Facharzt Voraussetzung für eine W2-Berufung sein und kann Verantwortung für ein Teilge- biet übernommen werden, auch wenn kein Facharztzeugnis, aber eine hervorragende morphologische und wissenschaftliche Qualifikation hierfür vorliegen? Neue Positionen und Werdegänge an Universitätsinstituten sollten attraktive Alternativen für wissenschaftlich Interessierte bieten.

Bestes Beispiel dafür, dass diagnostische Professionaliät außerhalb des Facharztstandards bereits existiert und aus der Arbeit eines Universitätsinstitutes nicht mehr weg zu denken ist, sind die Naturwissenschaftler, Biologen und Biochemiker, die in vielen unserer Institute molekularpathologische Diagnostik vertreten und auch in der Forschung aktiv sind. Sie sind es auch, die wesentlich an der bei dieser Jahrestagung erfolgenden Neugründung der Arbeitgemeinschaft (AG) Molekularpathologie, die eine organübergreifende allgemeinpathologische und methodische Ausrichtung haben wird, beteiligt sind und so ein institutionalisiertes Forum für den wissenschaftlichen Austausch schaffen.

Aber der molekularpathologisch selbst forschende Morphologe, kann nicht völlig durch diese uns erfreulicherweise zuwachsende Verstärkung ersetzt werden, denn Pathologie wird noch lange Zeit eine primär morphologische Disziplin bleiben, und die zukünftigen Führungspersönlichkeiten in unserem Fach sollten nicht nur den Brückenschlag leben, sondern auch zumindest in einem Teilgebiet exzeptionelle Histopathologen sein. So können sie leicht die Reichweite einer hervorragenden morphologischen Diagnostik zu dem, was eine ergänzende molekulare Untersuchung vermag, in das richtige Verhältnis setzen. „Rooming-In“ von forschungsstarken Gruppen, die selbst keine morphologische Pathologie mehr betreiben, löst das Problem allenfalls vorübergehend - kaum werden jemals aus Untermietern richtige Familienmitglieder.

In einer Zeit, in der eine mechanistische Forschungsrichtung mit verschiedensten Mausmodellen dominiert, wird die pathologiespezifische Herangehensweise der Untersuchung von Fallserien nicht selten als rein „deskriptive“ Forschung abqualifiziert. Kompetitive Forschung zur allgemeinen Tumorpathologie in den aktuellen Fragen der miRNA, 
Epigenetik, Seneszenz und Tumorstammzellen findet zumeist außerhalb von $\mathrm{Pa}$ thologieinstituten statt, auch wenn es einigen von uns gelingt, hier mitzuhalten. Wo wir aber auf jeden Fall bestehen können, ist bei der Übertragung von der Allgemeinen in die Spezielle Tumorpathologie. Wichtig ist vor diesem Hintergrund die Einbindung der Pathologie in prospektive klinische Studien. Referenz- und Studienpathologie stärken die Forschungsaktivität an einzelnen Standorten, ohne die an anderen zu schwächen. Die Materialrekrutierung innerhalb von Studien ist daher von eminenter Wichtigkeit für eine forschungsstarke Pathologie. Ergebnisse, die auf diesem Weg erzeugt werden, bauen Aussagemöglichkeit und Position der Pathologie insgesamt aus, und die Weitergabe von Blockmaterial sollte daher zumindest innerhalb der Universitätspathologien als ein vitales Interesse des gesamten Faches verstanden werden. Von Seiten der Fachgesellschaft und des BV sollte alles getan werden, um die Materialrekrutierung zu erleichtern und etwaigen Bedenken entgegenzutreten. Jede klinische Tumorstudie, die mit dem Argument, „die schicken sowieso nicht", eine pathologische Begleitforschung ausschließt, ist eine Niederlage für die Pathologie im Ganzen.

Die 94. Jahrestagung der DGP versucht ohne geliehenen Glanz und tumorbiologische Highlights, die andere gesetzt haben, auszukommen, sondern will deutlich machen, was an molekularpathologischer Forschung in der Pathologie vorhanden ist und die spezielle Organpathologie erweitern wird. Mehr als stark frequentierte Fortbildungsveranstaltungen muss ein gut besuchter und von eigenen Beiträgen lebender wissenschaftlicher Kongress als Lebens- und Überlebenszeichen eines Faches angesehen werden. Insofern wünsche ich den Referenten viele Zuhörer und allen Besuchern spannende neue Einsichten und Diskussionen.

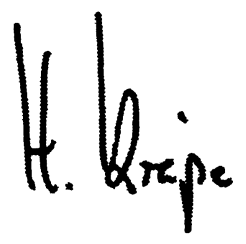

Hans H. Kreipe, Hannover

\section{Korrespondenzadresse}

Prof. Dr. H.H. Kreipe

Institut für Pathologie,

Medizinische Hochschule Hannover

Carl-Neuberg-Str. 1, 30625 Hannover

kreipe.hans@mh-hannover.de

Interessenkonflikt. Der korrespondierende Autor gibt an, dass kein Interessenkonflikt besteht. 